\title{
Implementation of a Newborn Clinical Decision Support Software (NoviGuide) in a Rural District Hospital in Eastern Uganda: Feasibility and Acceptability Study
}

Mary Muhindo ${ }^{1,2^{*}}, \mathrm{MBChB}, \mathrm{MPH}$; Joshua Bress ${ }^{3 *}, \mathrm{MD}$; Rogers Kalanda ${ }^{2^{*}}, \mathrm{DMCH}$; Jean Armas ${ }^{3 *}$, MPH; Elon Danziger $^{3 *}$, MArch; Moses R Kamya ${ }^{4 *}$, MBChB, DPhil; Lisa M Butler ${ }^{{ }^{*}}$, MPH, DPhil; Theodore Ruel ${ }^{6 *}$, MD, DPhil

${ }^{1}$ UCSF Preterm Birth Initiative, University of California San Francisco School of Medicine, San Francisco, CA, United States

${ }^{2}$ Infectious Diseases Research Collaboration, Kampala, Uganda

${ }^{3}$ Global Strategies, Albany, CA, United States

${ }^{4}$ School of Medicine, Makerere University College of Health Sciences, Kampala, Uganda

${ }^{5}$ Institute for Collaboration on Health, Intervention and Policy, University of Connecticut, Storrs, CT, United States

${ }^{6}$ Department of Pediatrics, University of California, San Francisco, San Francisco, CA, United States

*all authors contributed equally

\section{Corresponding Author:}

Mary Muhindo, MBChB, MPH

UCSF Preterm Birth Initiative

University of California San Francisco School of Medicine

550 16th Street, 3rd Floor

San Francisco, CA, 94158

United States

Phone: 1788551502

Email: marymkakuru@gmail.com

\section{Abstract}

Background: Lack of trained health care workers and nonadherence to national guidelines are key barriers to achieving high-quality newborn care in health care facilities in low- and middle-income countries. Traditional didactic approaches addressing these barriers fail to account for high staff turnover rates and result in temporary behavior change. NoviGuide, a clinical decision support software designed to standardize neonatal care through point-of-care assessments, has the potential to align bedside practice to national guidelines in settings lacking subspecialty neonatal providers.

Objective: This study aims to determine the adaptation, adoption, feasibility, acceptability, and sustainability of NoviGuide and its impact on nurse-midwives' knowledge in a rural hospital in eastern Uganda.

Methods: This mixed methods observational study was guided by the Proctor framework. Experts reviewed the clinical content of NoviGuide to ensure fidelity to Uganda guidelines. We enrolled nurses and midwives providing newborn care at Tororo District Hospital, trained them on NoviGuide use, and followed them for 12 months. We assessed adoption, feasibility, acceptability, and sustainability by analyzing NoviGuide use data, comparing it with maternity registry data and administering the System Usability Scale (SUS) and the Center for Health Care Evaluation Provider Satisfaction Questionnaire. We compared the mean knowledge assessment score at baseline, 6 months, and 12 months using a two-tailed $t$ test.

Results: Five Ugandan experts suggested two minor changes to NoviGuide: the inclusion of an unsterile birth environment as an indication for empiric antibiotics and the addition of a reminder to follow-up with newborns with temperatures between $37.7^{\circ} \mathrm{C}$ and $37.9^{\circ} \mathrm{C}$. Of the 19 nurse-midwives enrolled in February 2017, 74\% (n=14) completed the follow-up in March 2018. The participants entered a total of 1705 assessments of varying newborn characteristics into NoviGuide throughout the day, evening, and night nursing shifts. The SUS score at the end of the study was very high (93.5, above the average of 68). Participants had a positive perception about NoviGuide, reporting that NoviGuide saved time (mean 5, SD 0) and prevented mistakes (mean 5, SD 0 ), and that they felt more confident in taking care of newborns when they used NoviGuide (mean 5, SD 0). Participants were highly satisfied with NoviGuide (mean 4.86, SD 0.36), although they lacked medical supplies and materials needed to follow NoviGuide recommendations (mean 3.3, SD 1.22). The participants' knowledge scores improved by a mean change of 3.7 (95\% CI 2.6-4.8) at 6 months and $6.7(95 \%$ CI 4.6-8.2) at 12 months $(P<.001)$. 
Conclusions: NoviGuide was easily adapted to the Uganda guidelines. Nurse-midwives used NoviGuide frequently and reported high levels of satisfaction despite challenges with medical supplies and high staff turnover. NoviGuide improved knowledge and confidence in newborn care without in-person didactic training. NoviGuide use has the potential to scale up quality newborn care by facilitating adherence to national guidelines.

(JMIR Mhealth Uhealth 2021;9(2):e23737) doi: 10.2196/23737

\section{KEYWORDS}

clinical decision support; neonatology; neonatal mortality; mHealth; mobile phone

\section{Introduction}

\section{Background}

In 2018, 2.5 million children died in their first 28 days of life worldwide, with the highest neonatal mortality rate observed in sub-Saharan Africa (28 per 1000 live births) [1]. The causes of death in the neonatal period are well known, including complications of prematurity; intrapartum complications; and infections, such as sepsis, pneumonia, and meningitis [2]. Neonatal care protocols, such as those described in the World Health Organization (WHO) Essential Newborn Care guidelines [3], can lead to significant improvements and comprise the core of current global efforts to reduce neonatal mortality [4-7]. However, lack of trained health care workers and nonadherence to national neonatal care clinical guidelines are key barriers to achieving high-quality newborn care in health care facilities in low- and middle-income countries (LMICs) [8]. Implementation of evidence-based protocols in LMICs has been hampered by significant challenges: in-person training does not reliably lead to changes in workplace behavior, medical errors are common, changes in practice are lost quickly without reinforcement, high staff turnover rate, and performance of health care providers is difficult to monitor [9-12].

Traditional approaches to implementing neonatal care clinical guidelines in LMICs are based on lectures, the distribution of educational material, and hands-on training; although these approaches can be effective for focused topics or procedures such as neonatal resuscitation [13,14], they are not well suited to multistep neonatal protocols. A key component of behavior change is immediate and consistent feedback. Health care providers learning neonatal resuscitation receive immediate feedback on their performance through a newborn's second-to-second response to care, whereas the degree to which a provider's care aligns to a complex neonatal protocol, such as an antibiotic prescription guideline, cannot anticipate a similar deliberate practice benefit [15]. In addition, although the performance of a hands-on procedure is relatively constant across diverse patients, the application of complex neonatal protocols requires that health care providers must often account for patient-specific factors and adjust for site-specific constraints [16].
Clinical decision support (CDS) software has the potential to enable health care providers to deliver complex medical protocols as responsive point-of-care assessments [17-21]. CDS aims to achieve desired quality aims through care standardization rather than relying on individual performance, while improving health care provider satisfaction by deploying medical protocols through a validated user interface. A key feature that distinguishes CDS from traditional didactic training is that it does not rely on the health care provider to summon previously learned content at the point-of-care, either from memory or by seeking a resource. Instead, CDS uses patient-specific factors, such as a patient's age, vital signs, and symptoms, and directs health care providers to potentially relevant medical protocols.

\section{NoviGuide}

Our team developed NoviGuide (Global Strategies) [22], a tablet-based CDS software, to optimize the facility-based care of newborns in LMICs by transforming complex neonatal disease-specific protocols into comprehensive patient assessments.

NoviGuide has 3 main sections: Resuscitation, My Patient, and Learning (Figure 1). The Resuscitation section contains an instructional three-dimensional animation depicting the steps of neonatal resuscitation (Multimedia Appendix 1). The My Patient section houses point-of-care CDS algorithms that guide health care providers through the initial assessment and daily care of newborns. The Learning section is a standard e-book with additional information on neonatal topics and videos from the Global Health Media Project [23] depicting physical signs, common newborn procedures, and breastfeeding positions. A menu bar includes an emergency button to directly access clinical guidance for the treatment of neonatal seizures and abdominal emergencies (Figure 1). We selected algorithms for the emergency section based on the need for rapid guidance and the ability to generate that guidance based solely on the current dosing weight. NoviGuide was created by the nonprofit organization Global Strategies [24] for widespread use in low-resource settings. Global Strategies built the core software and content of NoviGuide and partnered with Plexus Medical Arts [25] to design the resuscitation video. The Global Health Media Project allowed the inclusion of its neonatal and breastfeeding films in the software. 
Figure 1. NoviGuide screenshots: Home page, NoviGuide clinical assessments, and Medication dosing instructions pages.

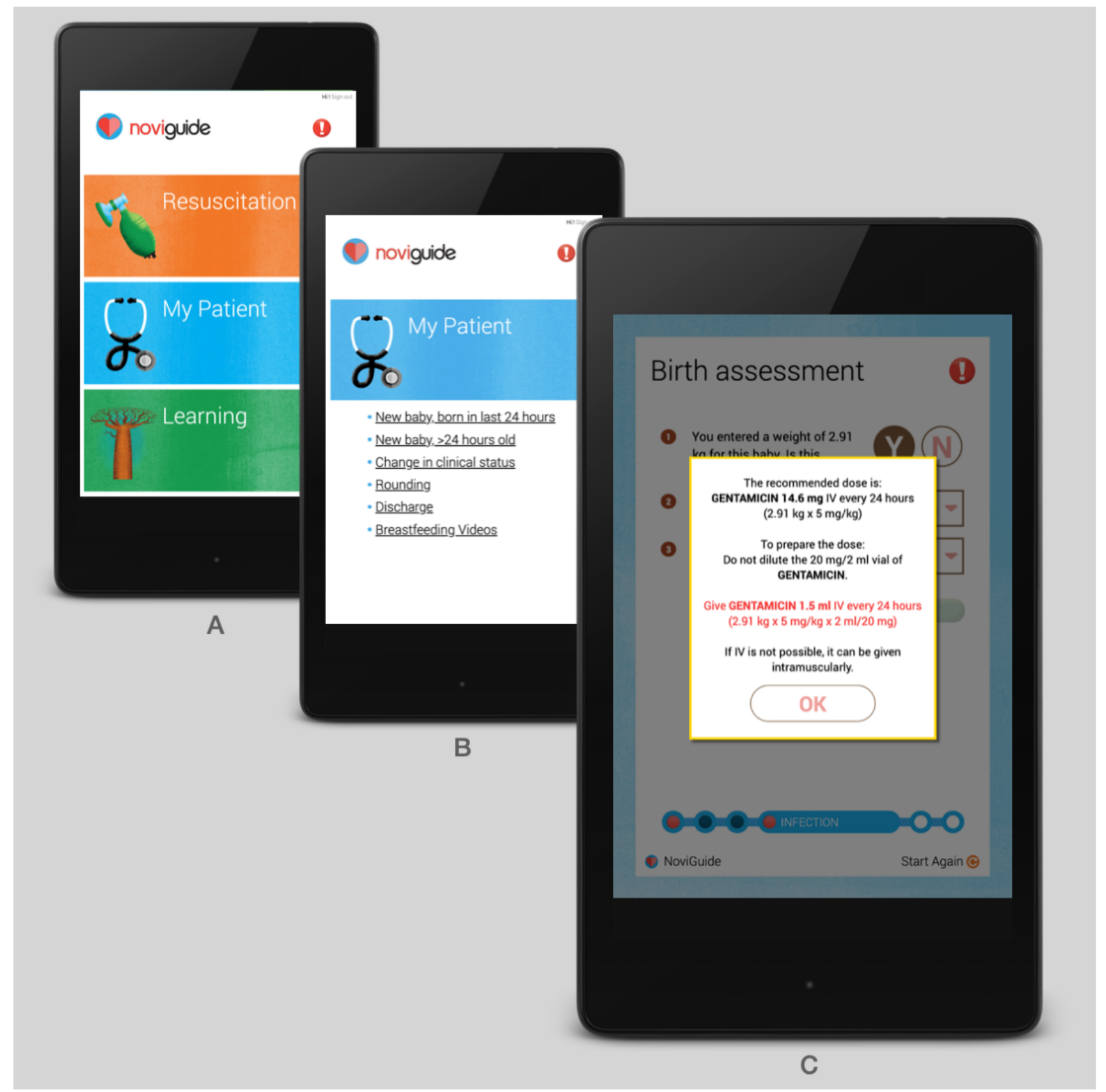

The My Patient CDS content includes 4 comprehensive clinical assessments to assist health care providers in the initial assessment; subsequent care; and discharge of well, sick, and preterm newborns. These assessments are named new baby more than 24 hours old or change in clinical status, rounding, and discharge (Figure 1). The assessments provide step-by-step prompts to guide the health care provider to enter data from the physical examination findings and key pieces of medical history (Multimedia Appendix 2). The assessments are dynamic, adding more questions in response to danger signs and alerting users to potential inconsistencies in their responses. NoviGuide then makes case-specific management recommendations derived from national clinical guidelines that are tailored to the newborn's weight, gestational age, day of life, clinical features, and available equipment. The My Patient assessments include numerous job facilitators to incentivize use, save time, and decrease medical errors (Multimedia Appendix 2). These job facilitators include preterm feeding calculators, medication and fluid calculators, guidance interpreting vital signs, and the generation of a printable summary. Responses to abnormal vital signs, infection risk stratification for antibiotic use, medication doses, and fluid calculations are provided automatically alongside any equations used to derive the recommendations (Figure 1).

When initiating an assessment, health providers are asked to indicate if the assessment is being completed on a real baby or just practicing (Multimedia Appendix 2). Users are often prompted to consider actions in NoviGuide's pathways that require resources. In numerous instances, users can indicate if they encounter a resource constraint related to the guidance, and these data are captured. NoviGuide works either offline or when connected to WiFi. All the information contained in the NoviGuide is present in the initial download. When NoviGuide is connected to the internet by $\mathrm{Wi}-\mathrm{Fi}$, data are automatically 
synchronized to a cloud-based database. NoviGuide is designed to work on Google Android, iOS, and FireOS platforms.

With this study, our aim is to describe the adaptation, adoption, feasibility, acceptability, and sustainability of NoviGuide use in a rural district hospital in eastern Uganda. We used a mixed methods observational study design among nurses and midwives in the context of newborn care. In addition, we analyzed the impact of NoviGuide use on the knowledge of nurse-midwives. The Proctor framework [26] guided the definition of implementation constructs with respect to the users and the local context.

\section{Methods}

\section{Adaptation of NoviGuide to Uganda Clinical Guidelines}

With the assistance of the Uganda Pediatrics Association, we recruited a team of 5 Ugandan experts to review the content of NoviGuide over a series of meetings between August and November 2016. The aim was to ensure the fidelity of NoviGuide to the Uganda neonatal care clinical guidelines and to refine the NoviGuide design to suit the Uganda local context [27]. The study team presented an overview and instructions on how to visualize and verify the content, order, and branch-point logic of NoviGuide's decision trees (Table 1). The study team also showed the experts how to evaluate NoviGuide's decision trees using test cases comprising sick and well neonatal scenarios. The study team highlighted specific content in which there are frequent variations in recommendations among national guidelines, including the preparation of medications and various pharmacologic diluents, the management of well-appearing newborns born to mothers with fevers and/or other sepsis risk factors, and the threshold at which a glucose level is considered low. For each clinical area, the experts viewed a series of videos that explained the clinical topic and how it manifests in NoviGuide's My Patient assessments. The study team gave each expert a tablet (Amazon Fire HD 8) loaded with NoviGuide version 1.6 to take home and instructed them to make notes of any recommendations. The tablets were returned following this activity. The Ugandan experts met for a second meeting for free discussion of their findings and to achieve consensus on a set of recommendations for Global Strategies.

Table 1. Description of content areas for Ugandan expert panel review.

\begin{tabular}{ll}
\hline Area & Description \\
\hline Content & $\begin{array}{l}\text { Verifying that the clinical information is consistent with national protocols. For example, the study team shows the expert panel } \\
\text { the temperature threshold where a newborn is considered febrile. The expert panel then votes to confirm or modify. } \\
\text { Order }\end{array}$ \\
$\begin{array}{l}\text { Verifying that the order of questions in each assessment is consistent with national protocols. For example, confirming that lines } \\
\text { of questioning concerning hypoglycemia should precede lines of questioning concerning the initiation of antibiotics. }\end{array}$ \\
$\begin{array}{l}\text { Branch-point } \\
\text { logic }\end{array}$ & $\begin{array}{l}\text { Reviewing how the software responds to user input and verifying that the response is consistent with national protocols. For example, } \\
\text { the study team shows the expert panel the alert message a user sees after entering a risk factor for infection. The expert panel then } \\
\text { votes to confirm or modify. }\end{array}$
\end{tabular}

\section{Study Setting}

We conducted an implementation study from February 2017 to March 2018 at Tororo District Hospital (TDH), a rural government-owned district hospital in eastern Uganda. TDH, a 200-bed facility, serves approximately 517,000 people, the majority of whom live in rural areas [28]. The hospital conducts about 360 births per month and receives sick newborns from the community, surrounding health centers, and private facilities including referrals from across the Kenya-Uganda border. One medical officer is assigned to work in the maternity ward. Nurses and midwives provide the majority of newborn care working in shifts of 2 to 3 providers. In addition, these same nurses and midwives provide care for laboring women and postpartum mothers and conduct all vaginal deliveries in a 6-bed labor suite. TDH has basic newborn care supplies, including bag-valve-masks, warming tables for resuscitation, intravenous (IV) supplies, and a standard country formulary for medications. At the time of the study, TDH did not have a dedicated neonatal care unit. Newborns requiring close nursing attention were cared for on 2 warming tables within the labor suite. Newborns requiring only intermittent IV antibiotic therapy were admitted by the medical team in the general postpartum ward with women requiring postnatal care.

\section{Enrollment and Training of Study Participants and Launch of Study}

We screened all the nurses and midwives working in the maternity ward at the TDH and enrolled them into the study in February 2017. Inclusion criteria for nurse-midwives included providing newborn care at $\mathrm{TDH}$, having current licenses to practice, and willingness to participate in the study. In addition, nurse-midwives had to have completed the WHO Integrated Management of Childhood Illnesses modules [29]. Participants exited the study if they were transferred by the hospital administration to another ward within the hospital, ceased employment at the hospital, or withdrew consent.

To recruit participants, we invited all nurse-midwives working at the TDH and their supervisors to attend an organized meeting in the hospital boardroom. The study team provided a brief introduction about the study, including NoviGuide and evaluation methods, and obtained written informed consent from the nurse-midwives who met the eligibility criteria. We asked the nurses and midwives who declined participation for their reasons. The medical superintendent, matron, and wards-in-charge were recruited as key informants in the study development; hospital leadership encouraged but did not mandate or require the use of NoviGuide. 
Following enrollment, the study participants attended a 3-hour training conducted by representatives of Global Strategies on how to use NoviGuide. Following the training, the participants created individual unique usernames and passwords to $\log$ in to the tablet and the NoviGuide software. The study team provided 7 tablets (Amazon Fire HD 8 tablet) loaded with NoviGuide in February 2017. The tablets were stored in a lockable wooden cabinet in the nurses' office in the labor suite. During the first week, the study team provided on-site technical support to troubleshoot technical issues. We followed the study participants through March 2018.

\section{Data Collection}

At baseline, participants completed a survey that included demographic data (age, sex, and level of education), years of clinical experience, experience using technology, and perceived challenges in caring for newborns at TDH.

The study participants also completed a questionnaire assessing basic knowledge in newborn care, including questions about the management of hypoglycemia, indications for antibiotics, management of the HIV-exposed infant, and the specific order of tasks in neonatal resuscitation. This questionnaire was then repeated at 6 and 12 months with modifications of the question order and variables, such as newborn weights, in the clinical scenarios (Multimedia Appendix 3).

Throughout the study period, the study team connected the tablets to a Wi-Fi network once per day to upload NoviGuide use data, stored in the tablet, onto a secure cloud-based database. NoviGuide use data were linked to the participants' unique study identification number. We compared the total number of assessments entered into the NoviGuide with the total number of births and admissions of newborns at the hospital during the study period. The study team instructed the participants to keep notes on any technical problems encountered during NoviGuide use in a study logbook or contact the study team by SMS, phone, or email for urgent concerns.

At 12 months, the participants completed 2 validated measures of software usability. We used the System Usability Scale (SUS) [30], consisting of 10 standard questions, where a statement is made and the respondent then indicates the degree of agreement or disagreement with the statement in a Likert scale format with responses 1 to 5 , where 1 represents strongly disagree and 5 represents strongly agree. We also used a provider satisfaction questionnaire adapted from the Center for Health Care Evaluation Provider Satisfaction Questionnaire (CHCE-PSQ) [31], which has 8 questions and the respondent then indicates the degree of agreement or disagreement with the statement in a Likert scale format with responses 1 to 5 . For questions 1 to 4 , response 1 represents poor and 5 represents excellent. Whereas for questions 5 to 8 , response 1 represents strongly disagree and 5 represents strongly agree. In addition, participants completed an end-of-study questionnaire containing 15 questions assessing perceived acceptability and feasibility of NoviGuide using a Likert scale of 1 to 5, where 1 represents strongly disagree and 5 represents strongly agree (Multimedia Appendix 3).

\section{Analysis}

We defined adoption as the measure of the initial uptake or intention to use the NoviGuide and measured it by reviewing the NoviGuide use data for (1) the different assessments made into the NoviGuide and how many of these were completed through to the summary page, (2) the time participants spent during the NoviGuide assessments, (3) NoviGuide use during the different nursing shifts (day, evening, and night), (4) whether participants accessed the NoviGuide's educational videos or reading materials and whether the participants used the NoviGuide for practice or with a real newborn, and (5) total NoviGuide assessments in relation to the total births and admissions at the hospital during the study period.

We defined acceptability as the measure of the participants' satisfaction with the various components of NoviGuide, including content, complexity, navigation, ease of use, and general experience using NoviGuide for newborn care, and measured it by (1) comparing the overall SUS score with an average score of 68, as described by John Brooke [30], and (2) determining the mean scores and SD of the questions in the CHCE-PSQ and end-of-study questionnaire. We calculated the overall SUS score by summing up the score contributions of each question and multiplying it by 2.5 .

We defined feasibility as the actual fit and the use of NoviGuide within the rural hospital context and measured it by reviewing the NoviGuide use data for (1) the characteristics of newborns cared for using NoviGuide and (2) whether the study participants indicated resource or health system constraints that could prevent the use of NoviGuide. We also measured feasibility by determining the mean scores and SD of questions 11 to 15 of the end-of-study questionnaire assessing the availability of medical supplies and materials needed to follow NoviGuide recommendations; time to use the NoviGuide; and support from colleagues, supervisors, and hospital administrators.

We defined sustainability as the extent to which NoviGuide use was maintained throughout the study period and the frequency and degree of technical problems preventing NoviGuide use. We measured use over the study period by individual users and collectively, across 100-day interval study periods (day 0-99, 100-199, 200-299, and 300-397).

We measured the impact of NoviGuide use on participant knowledge by comparing the mean knowledge assessment score at baseline with scores at 6 and 12 months using a paired $t$ test. We used Stata (version 16, StataCorp) for all statistical analyses. A $P$ value of $<.05$ was considered significant.

\section{Ethical Review}

The University of California San Francisco Committee on Human Research (16-19241), the Makerere University School of Biomedical Sciences (SB-352), and Uganda National Council for Science and Technology (IS 125) approved the study. All study participants provided written informed consent before participation in the study-related activities. 


\section{Results}

\section{Adaptation of NoviGuide to Uganda Clinical Guidelines}

The study team selected 4 Ugandan neonatologists and 1 Ugandan neonatal nurse as expert reviewers. The experts suggested 2 modifications to the decision trees. First, they recommended that birth in an unsterile environment should be added as a sepsis risk factor and that its presence should prompt a recommendation for empiric antibiotics. Second, they recommended that a specific pop-up message be generated for temperatures between $37.7^{\circ} \mathrm{C}$ and $37.9^{\circ} \mathrm{C}$ to alert users that the newborn was warm and they suggested that a follow-up temperature measurement be taken. Global Strategies incorporated these modifications into the NoviGuide decision trees.

Figure 2. Study flow diagram. TDH: Tororo District Hospital.

\section{Participant Characteristics and Follow-Up}

The study team screened 13 nurse-midwives and enrolled 12 nurse-midwives in February 2017 (Figure 2). One nurse declined to participate, citing that she was going to be away for further educational studies. Of the 12 participants in the initial enrollment group, 1 had a late start date of May 2017 because of maternity leave, and 5 participants were transferred to either other units within TDH or to other hospitals during the study period. In September 2017, following new hires at the maternity ward, the study team screened and enrolled an additional 7 nurse-midwives as replacements for those who had been transferred from the maternity ward. All 19 (100\%) study participants were female, with a mean age of 39 (SD 14) years (Table 2). Of the 19 participants, 11 (58\%) reported using a calculator on their phones to calculate medication dosages and fluid rates, $3(16 \%)$ used a handheld calculator, and 5 (26\%) performed the calculations mentally. All 19 participants reported that they believed that technology could help them in the care of newborns. Of the 19 enrolled participants, 14 (74\%) were followed up with until the end of the study period.

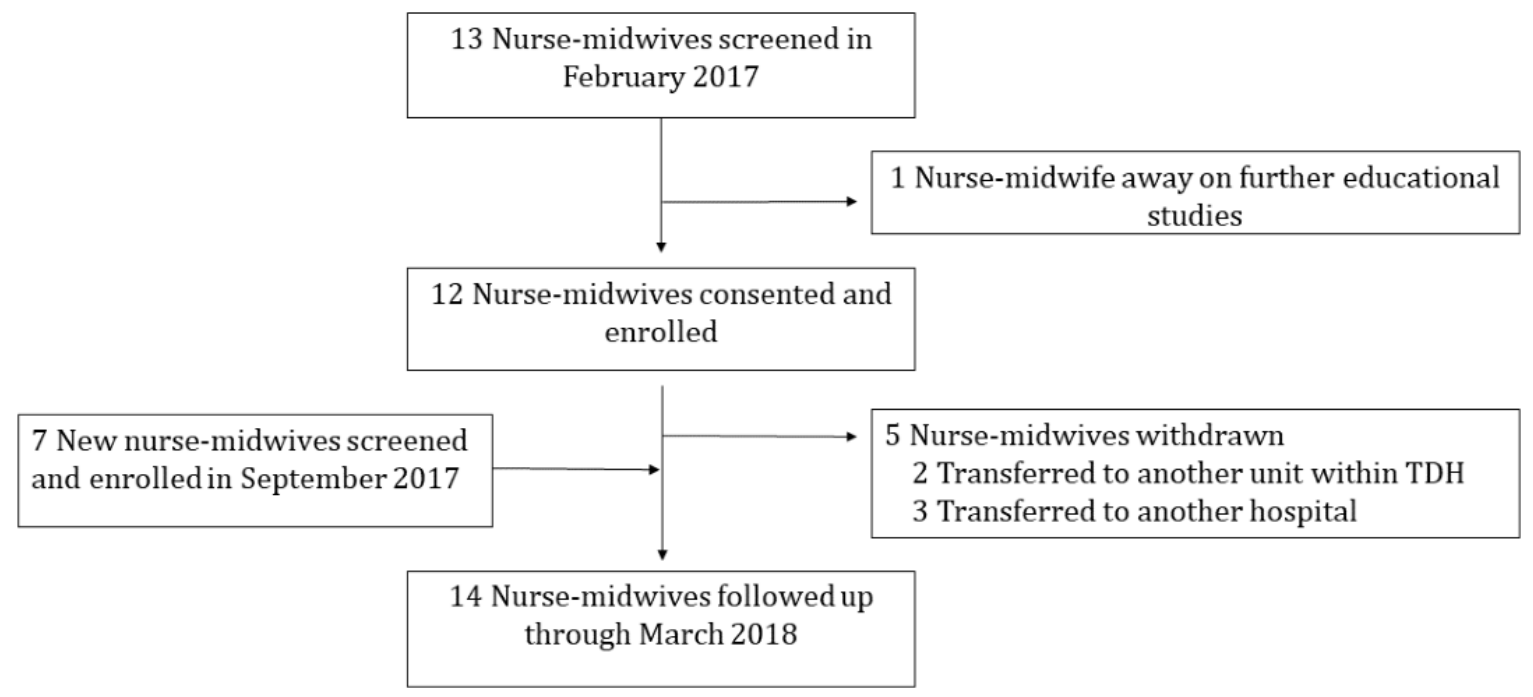


Table 2. Baseline demographics and participant characteristics $(n=19)$.

\begin{tabular}{ll}
\hline Characteristics & Values \\
\hline Female, $\mathrm{n}(\%)$ & $19(100)$ \\
Age (years), mean (SD) & $39(14)$
\end{tabular}

Highest educational level, n (\%)

Bachelor's

Registered nurse

Certified midwife

Work experience (years), n (\%)
$0-2$
3-10
$11-20$
$>21$

Devices owned personally, n (\%)

$\begin{array}{ll}\text { None } & 2(11) \\ \text { Home computer or laptop } & 1(5) \\ \text { Tablet } & 0(0) \\ \text { Smartphone } & 9(47) \\ \text { Ordinary phone } & 9(47)\end{array}$

How frequently do you access the internet, $n(\%)$

Never

Rarely or at least once a month

$6(32)$

Occasionally

Weekly

Daily at least once a day

Has technology made your life easier, n (\%)

Easier

No difference

Harder

Do you think technology can help you take care of babies, $n(\%)$

Yes

$19(100)$

No

$0(0)$

How do you describe yourself, $n(\%)$

I am the first to try something new

Before I try, I watch others try it and see if it fits my life 13 (68)

$\begin{array}{ll}\text { I am usually among the last to try something new } & 0(0)\end{array}$

How satisfied are you with the care of newborns at the Tororo District Hospital, $n$ (\%)
1 (least satisfied)
2
3
4
5
$5(28)$
6
$0(0)$
7 (most satisfied)
$0(0)$ 


\begin{tabular}{ll}
\hline Characteristics & Values \\
\hline How do you currently do the medication and fluid calculations, $\mathbf{n}(\%)$ & $3(16)$ \\
By handheld calculator & $11(58)$ \\
By calculator on the phone & $5(26)$ \\
I do them in my head & $14(74)$ \\
How do you decide when and which particular medication to give a sick baby, $\mathbf{n}(\boldsymbol{\%})$ & $12(63)$ \\
Check the World Health Organization chart at the maternity ward & $6(32)$ \\
Consult with the medical doctor & \\
I use my judgment and experience
\end{tabular}

\section{Adoption}

The study participants entered a total of 1705 assessments into NoviGuide over the study period. Of these 1705 assessments, $1412(82.82 \%)$ were completed through to the summary page. The most common completed entries were birth assessments with $65.93 \%$ (931/1412) assessments for new baby born in last 24 hours and 20.25\% (286/1412) assessments for new baby more than 24 hours old or change in clinical status (Figure 3), followed by discharge $6.51 \%$ (92/1412), rounding $5.59 \%$ (79/1412), seizure emergency $1.48 \%$ (21/1412), and abdominal emergency $0.21 \%$ (3/1412). Of the 293 uncompleted assessments, 161 (54.9\%) were for new baby born in last 24 hours, $81(27.6 \%)$ for new baby more than 24 hours old or change in clinical status, $32(10.9 \%)$ for rounding, $2(0.68 \%)$ for discharge, $15(5.1 \%)$ for seizure emergency, and $2(0.68 \%)$ for abdominal emergency.

The median time for a participant to complete assessments was 2.0 (IQR 1.0-5.0) min for new baby born in last 24 hours, 6.0 min (IQR 3.0-13.0) for new baby more than 24 hours old or change in clinical status, and 6.0 (IQR 4.0-10.5) $\mathrm{min}$ for rounding. In total, participants used the My Patient section for a total of 161 hours. NoviGuide was used frequently throughout the day, with 839 (49.2\%) assessments made during the day shift, $700(41.1 \%)$ during the evening shift, and $166(9.7 \%)$ during the night shift (Table 3).

All but 1 of the 19 study participants recorded entries into the NoviGuide. The mean (range) number of completed assessments per study participant was 90 (0-321). The participant without entries had been transferred to another hospital shortly after enrolling. Of the 1092 assessments of babies born within the last 24 hours, $68.13 \%$ (744/1092) were completed by only $26 \%$ $(5 / 19)$ study participants. Participants entered 46 practice cases, denoted by answering " $\mathrm{N}$ " (no) to the question, "Are you with a real baby? (Touch $\mathrm{N}$ if practicing)."

Data from the maternity register included 4704 admissions from February 1, 2017, to February 20, 2018. Of these, $97.55 \%$ (4589/4704) were identified as born at TDH, 2.32\% (109/4704) were born outside of TDH, and $0.13 \%$ (6/4704) entries did not specify the birth site. Six deaths $(0.13 \%)$ were recorded in the registry, and $0.64 \%$ (30/4704) newborns were transferred to a higher acuity facility. The registry, while noting whether the newborn was born at TDH, does not include the requisite data to determine whether the care encounter occurred immediately postpartum or upon return to the hospital following discharge.

Figure 3. NoviGuide use by assessment type.

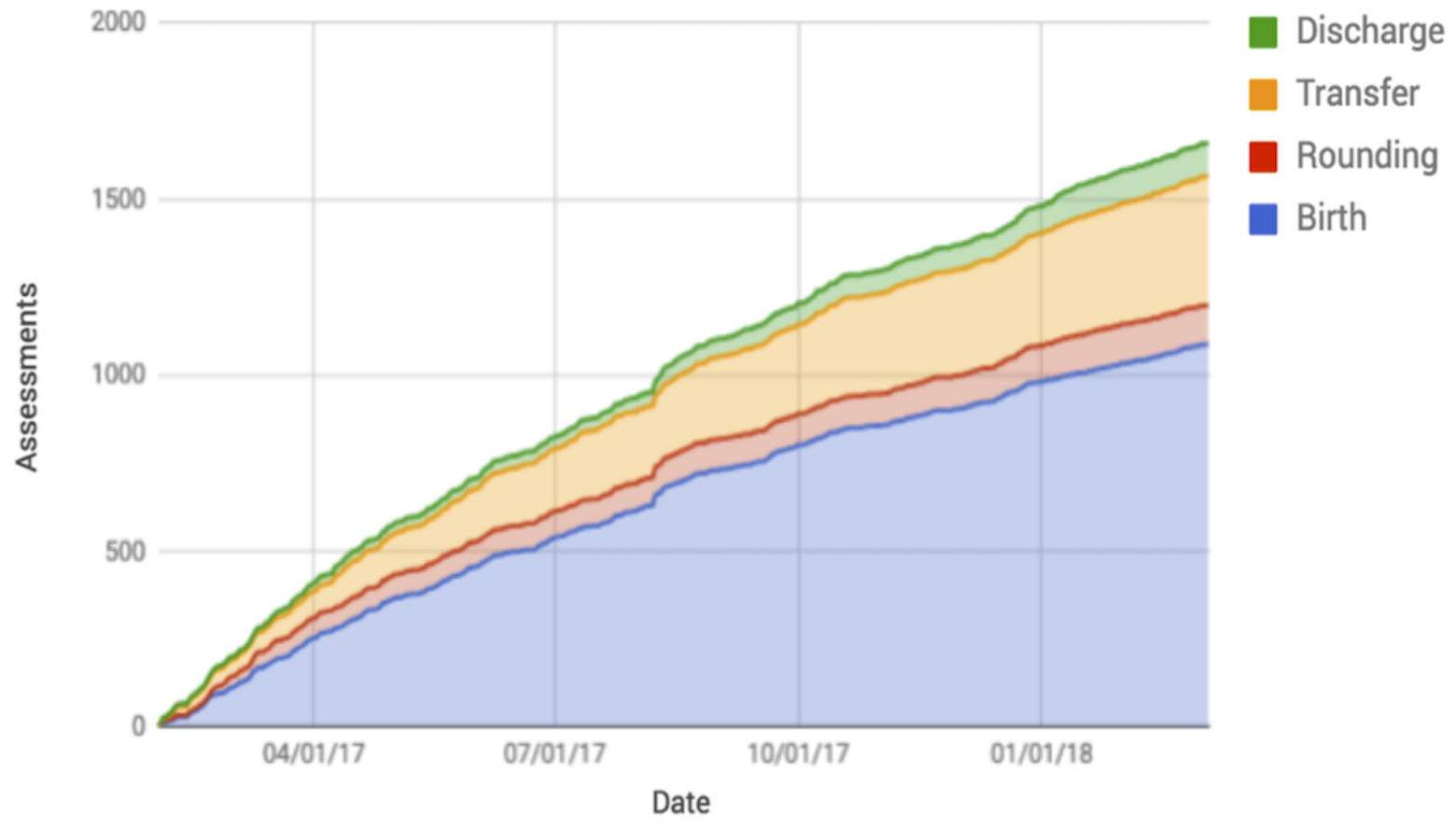


Table 3. NoviGuide use by work shifts and time of day.

\begin{tabular}{lllll}
\hline Assessment type & \multicolumn{2}{l}{ Assessments made during the different shifts and time of day } & \multicolumn{2}{c}{ Total } \\
& Day (8 AM to 2:59 PM) & Evening (3 PM to 7:59 PM) & Night (8 PM to 8 AM) \\
\hline New baby born in the last 24 hours, $\mathrm{n}$ & 529 & 451 & 112 & 40 \\
New baby more than 24 hours old, $\mathrm{n}$ & 176 & 151 & 3 & 367 \\
Rounding, $\mathrm{n}$ & 64 & 44 & 33 & 111 \\
Discharge, $\mathrm{n}$ & 54 & 1 & 0 & 94 \\
Abdominal emergency, $\mathrm{n}$ & 4 & 20 & 4 & 5 \\
Seizure emergency, $\mathrm{n}$ & 12 & 41.1 & 9.7 & 36 \\
Total $(\%)$ & 49.2 & & 100 \\
\hline
\end{tabular}

\section{Acceptability}

The overall SUS score at the end of the study was very high at 93.5 (Table 4) compared with the average score of 68 , as described by John Brooke [30]. The mean (SD) scores of all the questions in the CHCE-PSQ were more than 4 (out of a maximum of 5; Table 5). The participants reported high levels of satisfaction with the NoviGuide (mean 4.86, SD 0.36). The participants' perceptions about NoviGuide included the following: NoviGuide saved time (mean 5, SD 0), its information was useful (mean 4.79, SD 0.43), its information was easy to understand (mean 4.5, SD 0.52), the graphics were highly effective (mean 4.07, SD 0.47), and it could improve patient-nurse encounters (mean 5, SD 0).

In the end-of-study questionnaire (Table 5), the participants reported that NoviGuide helped them deliver better care and prevented them from making mistakes and that they felt more confident in taking care of newborns when they used the NoviGuide.

Table 4. Usability scores.

\begin{tabular}{lll}
\hline SUS $^{\mathrm{a}}$ & \multicolumn{1}{c}{${\text { Score, } \text { mean }^{\mathrm{b}} \text { (SD) }}^{\text {Converted }^{\mathrm{c}}}$} & 4 \\
\hline 1. I think that I would like to use the NoviGuide frequently. & $5(0)$ & $1.14(0.36)$ \\
2. I found the NoviGuide unnecessarily complex. & $4.7(0.46)$ & 3.9 \\
3. I thought the NoviGuide was easy to use. & $1.21(0.43)$ & 3.7 \\
4. I think that I would need the support of a technical person to be able to use NoviGuide. & $4.86(0.36)$ & 3.9 \\
5. I found the various functions in the NoviGuide were well integrated. & $1.36(0.74)$ & 3.6 \\
6. I thought there was too much inconsistency in the NoviGuide. & $4.57(0.65)$ & 3.6 \\
7. I would imagine that most people would learn to use the NoviGuide very quickly. & $1.21(0.80)$ \\
8. I found NoviGuide very cumbersome to use. & $5(0)$ & 3.8 \\
9. I felt very confident using the NoviGuide. & $1.86(1.23)$ \\
10. I needed to learn a lot of things before I could get going with the NoviGuide. & N/A ${ }^{\mathrm{d}}$ & 3.1 \\
Total converted mean scores $\times 2.5$ (overall SUS score) & 93.5
\end{tabular}

${ }^{\mathrm{a}}$ SUS: System Usability Scale.

${ }^{\mathrm{b}} 1$ : strongly disagree, 2 : somewhat disagree, 3 : neutral or no opinion, 4: somewhat agree, and 5: strongly agree.

${ }^{\mathrm{c}}$ For items 1, 3, 5, 7, and 9, the converted score is the mean score minus 1. For items 2, 4, 6, 8, and 10, the converted score is 5 minus the mean score. ${ }^{\mathrm{d}} \mathrm{N} / \mathrm{A}$ : not applicable. 
Table 5. Mean scores of the Center for Health Care Evaluation Provider Satisfaction Questionnaire and the end-of-study questionnaire.

\begin{tabular}{|c|c|}
\hline Questionnaires & Value, mean (SD) \\
\hline \multicolumn{2}{|l|}{ Center for Health Care Evaluation Provider Satisfaction Questionnaire ${ }^{a}$} \\
\hline 1. How useful is the information provided in the NoviGuide? & $4.79(0.43)$ \\
\hline 2. How easy is it to understand the information in the NoviGuide? & $4.5(0.52)$ \\
\hline 3. How effective are the graphics in NoviGuide? & $4.07(0.47)$ \\
\hline 4. What is your general satisfaction with the NoviGuide? & $4.86(0.36)$ \\
\hline 5. The NoviGuide could improve patient-nurse encounters & $5(0)$ \\
\hline 6. The NoviGuide saved me time & $5(0)$ \\
\hline 7. I would use it regularly in the clinic or hospital & $4.71(0.47)$ \\
\hline 8. I would recommend that other nurses use this tool & $5(0)$ \\
\hline \multicolumn{2}{|l|}{ Acceptability: end-of-study questionnaire ${ }^{b}$} \\
\hline 1. The NoviGuide helped me deliver better care to newborns & $5(0)$ \\
\hline 2. The NoviGuide prevented me from making a mistake while providing care to newborns & $5(0)$ \\
\hline 3. The NoviGuide improved my documentation on newborns and mothers & $4.79(0.43)$ \\
\hline 4. I was proud to use the NoviGuide & $4.93(0.27)$ \\
\hline 5. I feel more confident taking care of newborns when I use the NoviGuide & $5(0)$ \\
\hline 6. I think that using NoviGuide made a good impression on parents of the newborns I have seen & $4.71(0.61)$ \\
\hline 7. I think that using NoviGuide made a good impression on other parents in the community & $4.43(0.65)$ \\
\hline 8. I think that NoviGuide improved newborn care at my hospital & $4.93(0.27)$ \\
\hline 9. I think that using the NoviGuide to deliver newborn care at other hospitals is a positive idea & $5(0)$ \\
\hline 10. I think that NoviGuide is an important part of meeting my needs in caring for newborns & $5(0)$ \\
\hline \multicolumn{2}{|l|}{ Feasibility: end-of-study questionnaire } \\
\hline 11. I had the medical supplies and materials needed to follow NoviGuide recommendations & $3.36(1.22)$ \\
\hline 12. I had enough time to use the NoviGuide & $4.57(0.65)$ \\
\hline 13. My colleagues supported my use of the NoviGuide & $4.71(0.47)$ \\
\hline 14. My supervisor and the hospital administration supported my use of the NoviGuide & $4.71(0.47)$ \\
\hline 15. Technical support was always available for any difficulties I had with the NoviGuide & $4.93(0.27)$ \\
\hline
\end{tabular}

${ }^{\mathrm{a}}$ For Center for Health Care Evaluation Provider Satisfaction Questionnaire questions 1 to 4: 1, poor; 2, fair; 3, good; 4, very good; and 5, excellent; and for questions 5 to 8 : 1 , strongly disagree; 2 , somewhat disagree; 3 , neutral or no opinion; 4, somewhat agree; and 5 , strongly agree.

${ }^{\mathrm{b}}$ For the end-of-study questionnaire: 1 , strongly disagree; 2 , somewhat disagree; 3 , neutral or no opinion; 4 , somewhat agree; and 5 , strongly agree.

\section{Feasibility}

Study participants initiated and completed assessments on both well-appearing and ill-appearing newborns with diverse clinical characteristics (Table 6). Of the 1092 assessments for new baby born in last 24 hours, $29.21 \%$ (319/1092) were sick appearing, $24.82 \%$ (271/1092) had difficulty breathing, $19.51 \%(213 / 1092)$ weighed under $2.5 \mathrm{~kg}, 12.18 \%$ (133/1092) were born preterm, and $38.00 \%$ (415/1092) had at least one abnormal vital sign. Of the 367 assessments for new baby more than 24 hours old or change in clinical status, $77.4 \%(284 / 367)$ were sick appearing, $27.5 \%$ (101/367) had difficulty breathing, $53.1 \%$ $(195 / 367)$ had at least one abnormal sign, 30.2\% (111/367) weighed under $2.5 \mathrm{~kg}, 16.1 \%$ (59/367) were born preterm, and $59.9 \%(220 / 367)$ had antibiotics calculated during the assessment.
Rounding assessments included $6.51 \%(111 / 1705)$ of the total 1705 assessments entered into NoviGuide. Of these, $77.5 \%$ (86/111) were for term baby needing treatment, $18.0 \%$ (20/111) for preterm baby, and 4.5\% (5/111) for term baby.

In a number of instances, participants working in the same maternity ward reported varying resource capabilities. In 738 assessments where participants were guided to check a glucose level, $46.3 \%$ (342/738) reported that a glucometer was available and entered a level, whereas 53.7\% (396/738) responded Cannot test. Of the 264 completed assessments where a newborn had respiratory distress, participants indicated that a regular nasal cannula was available in $98.5 \%$ (260/264) assessments, whereas in $1.5 \%(4 / 264)$ assessments, participants indicated that there was no treatment available. Concerning the availability of IV fluids, participants indicated a desire to calculate IV fluid doses 
or rates in 328 assessments but indicated they could not give IV fluids in 20 assessments $(6.1 \%)$.

In the end-of-study questionnaire, participants responded with a mean score of 3.3 (SD 1.22) when asked whether they had the requisite resources to follow NoviGuide's recommendations
(Table 5). The participants' mean scores for the following questions were all above 4.5: (1) Was there enough time to use NoviGuide? (2) Was there support from their colleagues, supervisors, and hospital administration? and (3) Was technical support readily available?

Table 6. Characteristics of newborns entered into NoviGuide.

\begin{tabular}{lll}
\hline Newborn characteristics & $\begin{array}{l}\text { New baby born in the last 24 hours (n=1092), per- } \\
\text { cent birth assessments, } \mathrm{n}(\%)\end{array}$ & $\begin{array}{l}\text { New baby more than 24 hours old (n=367), percent } \\
\text { birth assessments, } \mathrm{n}(\%)\end{array}$ \\
\hline Sick appearing & $319(29.21)$ & $284(77.4)$ \\
Difficulty in breathing & $271(24.82)$ & $101(27.5)$ \\
Weight under $2.5 \mathrm{~kg}$ & $213(19.51)$ & $59(16.1)$ \\
Preterm (<37 weeks) & $133(12.18)$ & $195(53.1)$ \\
Abnormal vital signs ${ }^{\mathrm{a}}$ & $415(38.00)$ & $12(3.3)$ \\
HIV exposed & $55(5.04)$ & N/A ${ }^{\mathrm{b}}$ \\
Maternal fever & $70(6.41)$ & N/A \\
Foul smelling amniotic fluid & $109(9.98)$ & N/A \\
Born in an unsterile environment & $51(4.67)$ & $220(59.9)$ \\
Antibiotics calculated during assessment & $440(40.29)$ &
\end{tabular}

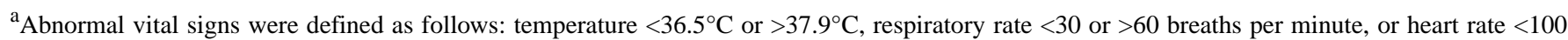
beats per minute or $>160$ beats per minute.

${ }^{\mathrm{b}} \mathrm{N} / \mathrm{A}$ : not applicable.

\section{Sustainability}

Although NoviGuide continued to be used regularly throughout the study (Figure 4), use declined with time. Study participants made $35.36 \%(603 / 1705)$ assessments on days 0-99 and then
$27.74 \%$ (473/1705), $18.82 \%$ (321/1705), and $18.06 \%$ (308/1705) assessments over the subsequent 100-day intervals. There were only 3 instances of minor technical issues; the only technical issue reported was screen freezing, which was easily resolved by either the participant or the study team.

Figure 4. NoviGuide use by user. Individual study participants had unique identification numbers starting with NG, followed by the number, for example, NG01.

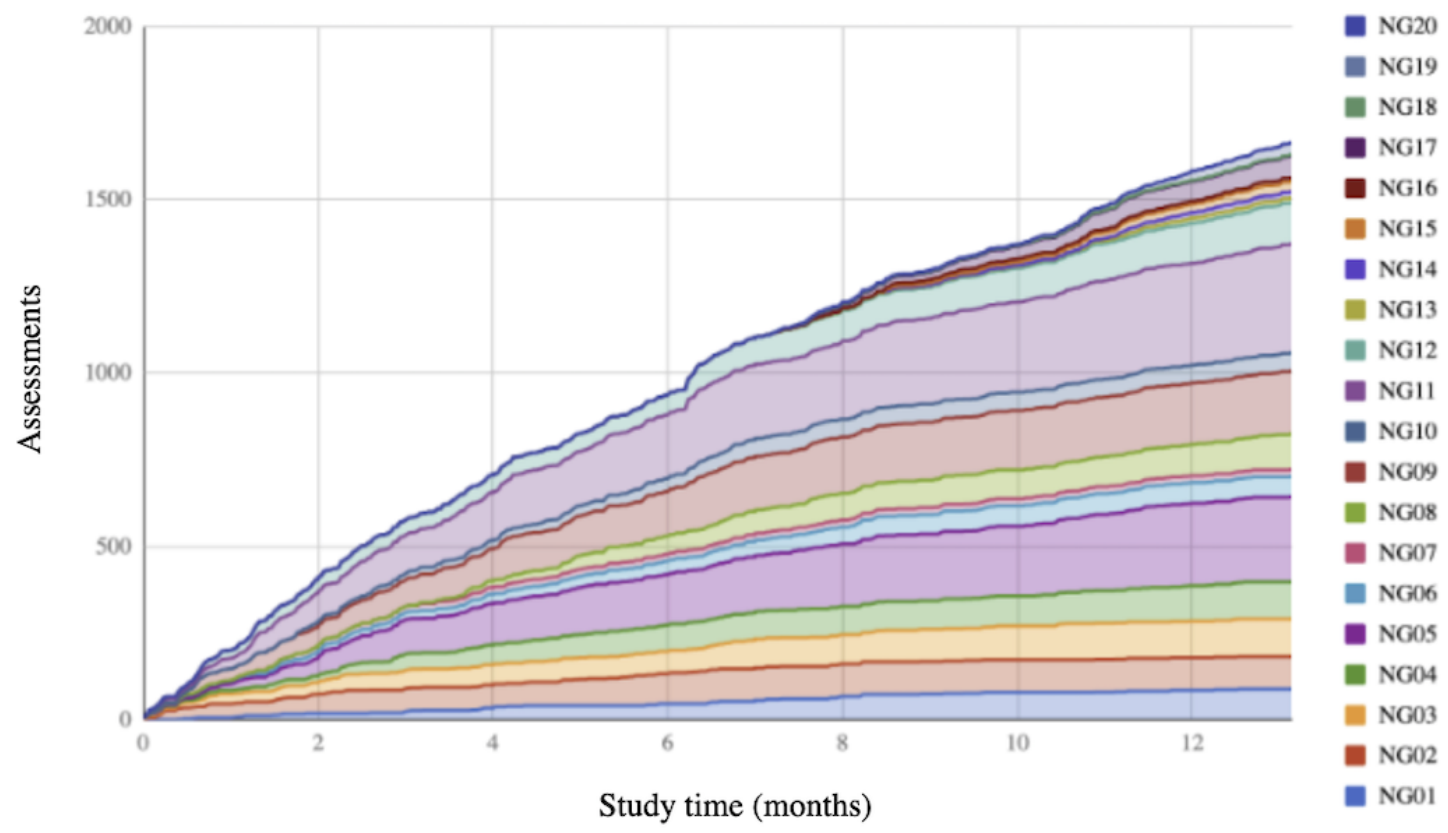




\section{Impact on Participant Knowledge}

The results from the knowledge assessment questionnaires demonstrated significant improvement in basic newborn care knowledge over time. Among the 18 participants who were assessed at 6 months, scores increased from a mean of 10.4 to 14.1, reflecting a mean change of 3.7 (95\% CI 2.6-4.8; $P<.001)$ points. Among the 8 patients who were assessed at 12 months, the mean change from baseline was 6.7 (95\% CI 5.07-8.31; $P<.001)$.

\section{Discussion}

\section{Principal Findings}

NoviGuide was easily adapted to Uganda clinical guidelines, and its implementation in a rural district hospital was feasible and acceptable to nurse-midwives caring for newborns. The nurse-midwives used NoviGuide across a range of clinical scenarios, reported high levels of satisfaction with the software, and reported that it significantly improved their knowledge of newborn care. This study adds to the growing evidence that CDS software designed for facility-based health care workers delivering complex inpatient care can increase the use of national clinical guidelines in LMICs [32-35].

There are a number of features that distinguish NoviGuide from previously reported neonatal CDS software designed for LMICs [32,36-38]. NoviGuide converts guideline documents into patient-specific guidance, providing contextual drug dosing and cross-referencing diagnoses with vital sign inputs. Although other CDS apps only provide users with treatment guidance for a pathology the user has selected, NoviGuide not only provides treatment guidance but also prompts users to consider pathologies based on patient-specific inputs. Finally, NoviGuide is not a medical record by design and therefore does not require patient identification inputs. This makes the time required to complete an assessment through NoviGuide significantly shorter.

Ugandan experts suggested only two minor modifications to NoviGuide's decision trees. Although the suggested additions are not explicitly detailed in the Uganda clinical guidelines, they do align with the national strategy to reduce deaths from newborn infections and with general standards of care. The paucity of modifications suggests that NoviGuide was well aligned to the Ugandan local context and suggests that there may be similar ease of adaptation in other countries where national guidelines are based on WHO recommendations on newborn health [36]. This finding has implications for scalability, as it suggests that algorithm templates with a few configurable settings may be acceptable in a wide range of health systems.

A key finding is that nurse-midwives had very high levels of satisfaction with NoviGuide; participants reported that the NoviGuide saved time, that they would recommend it to other nurses, and that they were even proud to use it. These findings highlight the potential of CDS as a delivery system for implementing complex clinical protocols. CDS-enabled functionalities, such as automated drug dose calculations, combined with a streamlined and attractive user interface, may confer a benefit on the user separate from that acquired by adhering to a specific clinical standard. Interestingly, these high levels of satisfaction with the NoviGuide persisted despite evidence that participants did not use it on every baby, there was wide variation in use, and there was an overall decrease in use over time. There are a number of possible explanations for the wide variation in use among participants, including differences in hours worked per individual, role within the ward, and planned absences. It is possible that autonomy in using, or not using, NoviGuide contributed to overall satisfaction with the software; NoviGuide may have been time saving because participants could self-select when they wanted to use it. Regardless, as in previous studies on CDS [20,37,38], our study demonstrates that the deployment of CDS in a way that does not mandate use will not capture all patients.

Participants improved their knowledge scores over the study period, even as they only rarely engaged with the parts of NoviGuide intended for self-directed learning outside of clinical care. This finding suggests that rather than becoming dependent on CDS to the detriment of internalized knowledge, CDS can improve provider knowledge through exposure. The finding that participants visited the self-directed learning section only 14 times over the study period requires additional qualitative investigation. One possibility is that health care providers form an early perception of the software as either a point-of-care software or a continuing education software, but not both.

A potentially important finding was that NoviGuide's use data captured entries where participants working at the same hospital reported that they had different resources to treat patients. This finding has potentially important implications, as it suggests that CDS could be used to identify instances where either an individual or system barrier prevents available resources from being used. The same data, if transmitted frequently, could facilitate the rapid identification of resource gaps, such as the stock out of drugs or malfunction of a previously functioning medical equipment.

\section{Limitations}

We acknowledge several limitations of our study. The newborn clinical characteristics entered into NoviGuide were not corroborated by reference to clinical charts or direct observation. Some of the investigators performing this evaluation were the designers of this tool, raising the possibility of bias; additional evaluation of our tool at a later stage in development could be informative. The lack of rolling enrollment may have influenced our adoption measurements, as new staff in the maternity ward began work before being enrolled in the study, resulting in a period where these staff observed NoviGuide in use but could not use it themselves. We also lack data to draw conclusions regarding the resource availability discrepancies identified through NoviGuide use. Specifically, we cannot determine whether these variances corresponded to a lack of provider comfort in using the resource, lack of access, equipment malfunction, or other causes. Finally, the Likert scale, which is commonly used to evaluate software acceptability and feasibility, is an imperfect tool and can result in response style bias [39]. 


\section{Conclusions}

A CDS software for neonatal health care providers can be an alternative method for implementing complex neonatal protocols in LMICs and may improve upon, and complement, the standard didactic approach because of its ability to couple clinical protocols with job aide functionalities. The NoviGuide software was easily adapted to Uganda neonatal care clinical guidelines, was used across a range of clinical scenarios, resulted in high levels of satisfaction, and significantly improved knowledge among nurse-midwives. Although CDS is not a solution for all the training needs of a health care workforce in LMICs, it may be the optimal choice for content that is complex, not easily retained or applied, and for which immediate performance feedback is not possible.

\section{Acknowledgments}

The authors would like to acknowledge the 5 Ugandan experts for their participation in the adaptation of NoviGuide to the Uganda neonatal care clinical guidelines: Dr Jolly Nankunda, consultant pediatrician, Mulago National Referral Hospital; Dr Victoria Nakibuuka, neonatologist, Nsambya Hospital; Dr Harriet Nambuya, consultant pediatrician, Jinja Regional Referral Hospital; Dr Tom Ediamu, consultant pediatrician, Hoima Regional Referral Hospital; and Damalie Mwogererwa, neonatal nurse, Mulago National Referral Hospital. The authors also acknowledge Dr Rebecca Nantada, the president of Uganda Pediatrics Association, and Prof Peter Waiswa, School of Public Health, Makerere University College of Health Sciences, for their guidance and support. The authors are grateful to the nurse-midwives for taking part in the study and to Dr Ochar Thomas, the medical superintendent, TDH, for the generous support. This study was supported by the East Africa Preterm Birth Initiative (PTBi), a multiyear, multicountry effort generously funded by the Bill \& Melinda Gates Foundation. The funders of the study had no role in data collection, analysis, interpretation, writing of this manuscript, or discussion to submit for publication.

\section{Authors' Contributions}

TR, LB, and JB conceived and designed the study. MM, JA, and KR designed the data collection forms. MM and KR collected, cleaned, and analyzed the data. TR, LB, and MK provided technical and mentorship support for MM, a research fellow with University of California San Francisco PTBi. JB and ED developed NoviGuide. All the coauthors critically revised the manuscript and approved the final draft before submission.

\section{Conflicts of Interest}

The following authors work with Global Strategies, the organization that developed NoviGuide: JB as President, ED as Director of Software Design and Development, and JA works as a Program Coordinator. JB, ED, and JA did not participate in the data collection process. TR is a member of the board of directors of Global Strategies and advised in the development of NoviGuide. However, he receives no financial compensation from the organization or is in any other way related to NoviGuide; neither he nor his family members have any potential financial benefit from this manuscript's publication. The remaining authors declare no conflicts of interest.

\section{Multimedia Appendix 1}

NoviGuide resuscitation video. [PPTX File, 32148 KB-Multimedia Appendix 1]

\section{Multimedia Appendix 2}

NoviGuide step-by-step prompts.

[PPTX File, 43816 KB-Multimedia Appendix 2]

\section{Multimedia Appendix 3}

System Usability Scale and provider satisfaction questionnaire adapted from the Center for Health Care Evaluation Provider Satisfaction Questionnaire, the end-of-study questionnaire, and the knowledge assessment questionnaire.

[DOC File, 136 KB-Multimedia Appendix 3]

\section{References}

1. Newborns: improving survival and well-being. World Health Organization. 2020 Sep 19. URL: https://www.who.int/ news-room/fact-sheets/detail/newborns-reducing-mortality [accessed 2021-01-16]

2. WHO_CHERG methods and data sources for child causes of death 2000-2011. Department of Health Statistics and Information Systems (WHO) and WHO-UNICEF Child Health Epidemiology Reference Group (CHERG). URL: https:/ /www.who.int/healthinfo/statistics/ChildCOD method.pdf [accessed 2021-01-16] 
3. Pocketbook of Hospital Care for Children - Guidelines for the management of common childhood illness. 2nd Edition. World Health Organization. 2013. URL: https://apps.who.int/iris/bitstream/handle/10665/81170/9789241548373 eng. pdf?sequence=1 [accessed 2021-02-09]

4. Chomba E, McClure EM, Wright LL, Carlo WA, Chakraborty H, Harris H. Effect of WHO newborn care training on neonatal mortality by education. Ambul Pediatr 2008;8(5):300-304 [FREE Full text] [doi: 10.1016/j.ambp.2008.04.006] [Medline: 18922503]

5. Haws RA, Thomas AL, Bhutta ZA, Darmstadt GL. Impact of packaged interventions on neonatal health: a review of the evidence. Health Policy Plan 2007 Jul;22(4):193-215. [doi: 10.1093/heapol/czm009] [Medline: 17526641]

6. Lee ACC, Cousens S, Wall SN, Niermeyer S, Darmstadt GL, Carlo WA, et al. Neonatal resuscitation and immediate newborn assessment and stimulation for the prevention of neonatal deaths: a systematic review, meta-analysis and Delphi estimation of mortality effect. BMC Public Health 2011 Apr 13;11 Suppl 3:S12 [FREE Full text] [doi:

10.1186/1471-2458-11-S3-S12] [Medline: 21501429]

7. Darmstadt GL, Shiffman J, Lawn JE. Advancing the newborn and stillbirth global agenda: priorities for the next decade. Arch Dis Child 2015 Feb;100 Suppl 1:13-18. [doi: 10.1136/archdischild-2013-305557] [Medline: 25613960]

8. Dickson KE, Simen-Kapeu A, Kinney MV, Huicho L, Vesel L, Lackritz E, Lancet Every Newborn Study Group. Every Newborn: health-systems bottlenecks and strategies to accelerate scale-up in countries. Lancet 2014 Aug 02;384(9941):438-454. [doi: 10.1016/S0140-6736(14)60582-1] [Medline: 24853600]

9. Bang A, Patel A, Bellad R, Gisore P, Goudar SS, Esamai F, et al. Helping Babies Breathe (HBB) training: What happens to knowledge and skills over time? BMC Pregnancy Childbirth 2016 Nov 22;16(1):364 [FREE Full text] [doi: 10.1186/s12884-016-1141-3] [Medline: 27875999]

10. Mbwele B, Reddy E, Reyburn H. A rapid assessment of the quality of neonatal healthcare in Kilimanjaro region, northeast Tanzania. BMC Pediatr 2012 Nov 21;12:182 [FREE Full text] [doi: 10.1186/1471-2431-12-182] [Medline: 23171226]

11. Reisman J, Arlington L, Jensen L, Louis H, Suarez-Rebling D, Nelson BD. Newborn Resuscitation Training in Resource-Limited Settings: A Systematic Literature Review. Pediatrics 2016 Aug;138(2) [FREE Full text] [doi: 10.1542/peds.2015-4490] [Medline: 27388500]

12. Kalyan G, Vatsa M. Neonatal nursing: an unmet challenge in India. Indian J Pediatr 2014 Nov;81(11):1205-1211. [doi: 10.1007/s12098-014-1567-4] [Medline: 25278279]

13. Ersdal HL, Vossius C, Bayo E, Mduma E, Perlman J, Lippert A, et al. A one-day. Resuscitation 2013 Oct;84(10):1422-1427. [doi: 10.1016/j.resuscitation.2013.04.005] [Medline: 23612024]

14. Kc A, Wrammert J, Nelin V, Clark RB, Ewald U, Peterson S, et al. Evaluation of Helping Babies Breathe Quality Improvement Cycle (HBB-QIC) on retention of neonatal resuscitation skills six months after training in Nepal. BMC Pediatr 2017 Apr 11;17(1):103 [FREE Full text] [doi: 10.1186/s12887-017-0853-5] [Medline: 28399847]

15. Ericsson KA, Krampe RT, Tesch-Römer C. The role of deliberate practice in the acquisition of expert performance. Psychological Review 1993;100(3):363-406. [doi: 10.1037/0033-295x.100.3.363]

16. Rowe AK, de Savigny D, Lanata CF, Victora CG. How can we achieve and maintain high-quality performance of health workers in low-resource settings? Lancet 2005;366(9490):1026-1035. [doi: 10.1016/S0140-6736(05)67028-6] [Medline: 16168785]

17. Karlsson LO, Nilsson S, Bang M, Nilsson L, Charitakis E, Janzon M. A clinical decision support tool for improving adherence to guidelines on anticoagulant therapy in patients with atrial fibrillation at risk of stroke: A cluster-randomized trial in a Swedish primary care setting (the CDS-AF study). PLoS Med 2018 Mar;15(3):e1002528 [FREE Full text] [doi: 10.1371/journal.pmed.1002528] [Medline: 29534063]

18. Goud R, van Engen-Verheul M, de Keizer NF, Bal R, Hasman A, Hellemans IM, et al. The effect of computerized decision support on barriers to guideline implementation: a qualitative study in outpatient cardiac rehabilitation. Int J Med Inform 2010 Jun;79(6):430-437. [doi: 10.1016/j.ijmedinf.2010.03.001] [Medline: 20378396]

19. Bright TJ, Wong A, Dhurjati R, Bristow E, Bastian L, Coeytaux RR, et al. Effect of clinical decision-support systems: a systematic review. Ann Intern Med 2012 Jul 03;157(1):29-43 [FREE Full text] [doi: 10.7326/0003-4819-157-1-201207030-00450] [Medline: 22751758]

20. Toth-Pal E, Wardh I, Strender L, Nilsson G. A guideline-based computerised decision support system (CDSS) to influence general practitioners management of chronic heart failure. Inform Prim Care 2008;16(1):29-39 [FREE Full text] [doi: 10.14236/jhi.v16i1.672] [Medline: 18534075]

21. Agarwal S, Perry HB, Long L, Labrique AB. Evidence on feasibility and effective use of mHealth strategies by frontline health workers in developing countries: systematic review. Trop Med Int Health 2015 Aug;20(8):1003-1014 [FREE Full text] [doi: 10.1111/tmi.12525] [Medline: 25881735]

22. NoviGuide. 2019 Oct 21. URL: https://www.noviguide.com/ [accessed 2021-01-16]

23. Global Health Media Project. 2019 Oct 21. URL: https://globalhealthmedia.org/ [accessed 2021-01-16]

24. Global Strategies. 2019 Oct 29. URL: https://www.globalstrategies.org/ [accessed 2021-01-16]

25. Plexus Medical Arts. 2019 Oct 21. URL: https://plexusart.com/ [accessed 2021-01-16] 
26. Proctor E, Silmere H, Raghavan R, Hovmand P, Aarons G, Bunger A, et al. Outcomes for implementation research: conceptual distinctions, measurement challenges, and research agenda. Adm Policy Ment Health 2011 Mar;38(2):65-76 [FREE Full text] [doi: 10.1007/s10488-010-0319-7] [Medline: 20957426]

27. Sundell K, Beelmann A, Hasson H, von Thiele Schwarz U. Novel Programs, International Adoptions, or Contextual Adaptations? Meta-Analytical Results From German and Swedish Intervention Research. J Clin Child Adolesc Psychol 2016;45(6):784-796. [doi: 10.1080/15374416.2015.1020540] [Medline: 25864716]

28. Uganda Demographic and Health Survey 2016. 2016. URL: https:/www.ubos.org/onlinefiles/uploads/ubos/pdf\%20documents/ Uganda DHS 2016 KIR.pdf [accessed 2021-01-16]

29. Documents on the Integrated Management of Childhood Illness (IMCI). World Health Organization. 2020 Jan 16. URL: https://www.who.int/maternal_child_adolescent/documents/imci/en/ [accessed 2021-02-09]

30. Brooke J. SUS-A quick and dirty usability scale. In: Jordan PW, Thomas B, McLelland I, Weerdmeester BA, editors. Usability Evaluation in Industry. London: CRC Press; 1996:189-194.

31. Kirkpatrick D. Techniques for evaluating training programs. In: Ely DP, Plomp T, editors. Classic Writings on Instructional Technology. Englewood CO: Libraries Unlimited Inc; 1996.

32. Crehan C, Kesler E, Nambiar B, Dube Q, Lufesi N, Giaccone M, et al. The NeoTree application: developing an integrated mHealth solution to improve quality of newborn care and survival in a district hospital in Malawi. BMJ Glob Health 2019;4(1):e000860 [FREE Full text] [doi: 10.1136/bmjgh-2018-000860] [Medline: 30713745]

33. Jensen C, McKerrow NH, Wills G. Acceptability and uptake of an electronic decision-making tool to support the implementation of IMCI in primary healthcare facilities in KwaZulu-Natal, South Africa. Paediatr Int Child Health 2020 Nov;40(4):215-226. [doi: 10.1080/20469047.2019.1697573] [Medline: $\underline{31779539]}$

34. Kawamoto K, Houlihan CA, Balas EA, Lobach DF. Improving clinical practice using clinical decision support systems: a systematic review of trials to identify features critical to success. BMJ 2005 Apr 02;330(7494):765 [FREE Full text] [doi: 10.1136/bmj.38398.500764.8F] [Medline: 15767266]

35. Adepoju IO, Albersen BJA, De Brouwere V, van Roosmalen J, Zweekhorst M. mHealth for Clinical Decision-Making in Sub-Saharan Africa: A Scoping Review. JMIR Mhealth Uhealth 2017 Mar 23;5(3):e38 [FREE Full text] [doi: 10.2196/mhealth.7185] [Medline: 28336504]

36. WHO recommendations on newborn health: guidelines approved by the WHO Guidelines Review Committee. World Health Organization. 2017. URL: https://apps.who.int/iris/handle/10665/259269 [accessed 2021-02-09]

37. Arts DL, Abu-Hanna A, Medlock SK, van Weert HCPM. Effectiveness and usage of a decision support system to improve stroke prevention in general practice: A cluster randomized controlled trial. PLoS One 2017;12(2):e0170974 [FREE Full text] [doi: 10.1371/journal.pone.0170974] [Medline: 28245247]

38. Mainous AG, Lambourne CA, Nietert PJ. Impact of a clinical decision support system on antibiotic prescribing for acute respiratory infections in primary care: quasi-experimental trial. J Am Med Inform Assoc 2013;20(2):317-324 [FREE Full text] [doi: 10.1136/amiajnl-2011-000701] [Medline: 22759620]

39. Paulhus D. Measurement and Control of Response Bias. Measures of Personality and Social Psychological Attitudes 1991:17. [doi: 10.1016/b978-0-12-590241-0.50006-x]

\section{Abbreviations}

CDS: clinical decision support

CHCE-PSQ: Center for Health Care Evaluation Provider Satisfaction Questionnaire

IV: intravenous

LMIC: low- and middle-income country

PTBi: Preterm Birth Initiative

SUS: System Usability Scale

TDH: Tororo District Hospital

WHO: World Health Organization

Edited by L Buis; submitted 21.08.20; peer-reviewed by J Li, R Carroll, $N$ Mohammad Gholi Mezerji, M K.; comments to author 06.10.20; revised version received 22.11.20; accepted 04.01.21; published 19.02.21

Please cite as:

Muhindo M, Bress J, Kalanda R, Armas J, Danziger E, Kamya MR, Butler LM, Ruel T

Implementation of a Newborn Clinical Decision Support Software (NoviGuide) in a Rural District Hospital in Eastern Uganda: Feasibility and Acceptability Study

JMIR Mhealth Uhealth 2021;9(2):e23737

URL: http://mhealth.jmir.org/2021/2/e23737/

doi: $10.2196 / 23737$

PMID: 33605886 
(C) Mary Muhindo, Joshua Bress, Rogers Kalanda, Jean Armas, Elon Danziger, Moses R Kamya, Lisa M Butler, Theodore Ruel. Originally published in JMIR mHealth and uHealth (http://mhealth.jmir.org), 19.02.2021. This is an open-access article distributed under the terms of the Creative Commons Attribution License (https://creativecommons.org/licenses/by/4.0/), which permits unrestricted use, distribution, and reproduction in any medium, provided the original work, first published in JMIR mHealth and uHealth, is properly cited. The complete bibliographic information, a link to the original publication on http://mhealth.jmir.org/, as well as this copyright and license information must be included. 